\title{
Effect on blood cholesterol level with increase in concentration of probiotics to Giriraja and Sakini chicken in Nepal
}

\author{
D Neupane ${ }^{1 *}$, DB Nepali ${ }^{2}$, NR Devkota ${ }^{2}$, MP Sharma² and IP Kadariya ${ }^{2}$
}

Affiliation: ${ }^{1}$ Nepal Agricultural Research Council, Swine and Avian Research Program, Khumaltar, Nepal; ${ }^{2}$ Agriculture and Forestry University (AFU), Chitwan, Nepal

\begin{abstract}
The chicken population is increasing and the meat is acceptable by most ethnic groups, these species are remarkable for the meat production in the country. Probiotic microorganisms have played significant role in lowering cholesterol which have been documented over the past two decades via various clinical trials. This Cholesterol lowering properties of microorganism have been proposed to deconjugate bile via production of bile salt hydrolase (BSH) associated with their cholesterol lowering potentials in prevention of hypercholesterolemia. Deconjugated bile salts are more hydrophobic than their conjugated counterparts, thus are less reabsorbed through the intestines resulting in higher excretion into the feces. Of total 16 replicate, T1R1-T1R4 were without probiotics whereas T2R1-T2R4, T3R1-T3R4, T4R1T4R4 were with probiotics $5 \%, 10 \%$ and $15 \%$ concentration Probiotics for Sakini male and female. Similary for Giriraja breed T5R1-T5R4, T6R1-T6R4, T7R1-T7R4 and T8R1-T8R4 were 0\%, 5\%, 10\% and $15 \%$ concentration of with Probiotics. There was comparatively degradation of Cholesterol in blood with increase in probiotics concentration via Cell Net Analyzer.
\end{abstract}

Key words: probiotic, cholesterol, Giriraja, Sakini

Bangladesh Animal Husbandry Association. All rights reserved.

Bang. J. Anim. Sci. 2017. 46 (4):225-229

\section{Introduction}

Poultry is one of the fastest growing segments of the agricultural sector in Nepal and produced chicken meat at 114,058 tonnes annually, while egg output amounts to 1.20 billion sets annually (CBS 2016). Sakini is indigenous breed of chicken and fetched high price for its egg and meat in the market, the low productivity of the breed could not meet the consumers demand (Bhurtel and Sah et al., 2000). Therefore, for upgrading indigenous breeds in rural areas, "Giriraja" a synthetic colored dual-purpose strain resemble local fowl introduced in 1970's in the country (Shrestha, 2000). Meat and egg from both breeds have high demand in market with its special taste. On other hand, several feed additives (as growth promoter) like synthetic hormone and antibiotics have been extensively used for enhancing poultry production but due to development of antibiotic resistant bacterial strains and residual effects of these feed additives in eggs and meat, they lead to various health hazards to consumers. Thus, probiotics has been introduced as alternative feed additives in poultry, which can be used in animal nutrition on a safe basis.
Probiotics have been defined as "live microorganisms which when administered in adequate amount confer a health benefit to the host"(Morelli and Capurso, 2012). However, intake of Probiotic lowers the risk of gastrointestinal diseases by stimulating the growth of beneficial microorganisms (Fuller, 1989; Chiang and Pan, 2012). The WHO has predicted that by 2030 , cardiovascular diseases will remain the leading causes of death, affecting approximately 23.6 million people around the World (WHO, 2009). It was reported that supplementation of probiotics may avert the use of cholesterol-lowering drugs in people with high cholesterol level profile (WHO, 2009). It had been proposed that the hypocholesterolemic effect of probiotics was due to the assimilation of cholesterol during growth, thus reducing the amount of cholesterol available for absorption in the intestine (Lye et al., 2009). However, effects of probiotics in Sakini and Giririaja chickens were not investigated yet, therefore the present study was conducted to know the concentration of blood cholesterol level with increasing level of probiotics added diet of Sakini and Giriraja chickens in Nepal. 


\section{Materials and Methods}

A total of 640 birds of two breeds (Sakini- 320 and Giriraja -320) with equal number of male and female of aged 100 days were selected and was divided into eight treatments and eight replication having 10 birds in each replication in $2 * 4 * 8$ factorial RCBD design. The basal feed was formulated and feed layer grower diet (L2$16 \%$ Cruide protein \& $2700 \mathrm{KcalME} / \mathrm{kg}$ ) for 26 days and then layer finisher (L3- 18\% Crude protein \& $2800 \mathrm{KcalME} / \mathrm{kg}$ ) diet unit the end of the experiment as in table 1 . Chicks were assigned into one of the following treatments: control (basal diet without added probiotics), and same basal diet supplemented with $0.5 \%$, $1 \%$ and $1.5 \%$ of poultry biosa were added to make the other treatments. Poultry biosa (Biosa Nepal Agriculural Pvt. Ltd) is multi-strain commercial preparation in liquid form (CFU/gm) that consist of Bifidobacterium lactis, Bifidobacterium longum, Lactobacillus acidophilus, Lactobacillus casei, Lactobacillus rhamnosus, Lactobacillus salivarius, Lactococcus lactis, Streptococcus thermophilus. The feed and water was provided adlibitum throughout the experiment. At the end of the experiment at 120 days, blood samples were taken randomly from one bird from each replication or each experimental unit. Thus, total 64 blood samples were collected from the venacutanea ulnaris. Before taking the blood sample birds were fasten for 12 hrs and no any medicine and vitamins were given for 24 hrs prior to the blood drawn. Whole blood was drawn into EDTA-containing tubes, centrifuged at $4{ }^{\circ} \mathrm{C}$ for $15 \mathrm{~min}$.

Table 1. Experimental diet fed to Sakini and Giriraja chickens during the experiment

\begin{tabular}{lrr}
\hline & $\begin{array}{r}\text { Grower } \\
\text { ration } \\
\text { (L2) }\end{array}$ & $\begin{array}{r}\text { Finisher } \\
\text { ration } \\
\text { (L3) }\end{array}$ \\
\hline Ingredients \% & & \\
\hline Maize & 44.00 & 40.00 \\
Soybean meal & 10.00 & 10.00 \\
Rice bran & 12.00 & 12.00 \\
Meat meal & 3.00 & 7.50 \\
Oil & 0.07 & 0.08 \\
Mineral & 1.23 & 1.00 \\
Salt & 1.00 & 0.50 \\
Mustard cake & 11.00 & 11.00 \\
Wheat grain & 16.00 & 16.50 \\
Lysine & 0.10 & 0.10 \\
Methionine & 0.10 & 0.10 \\
DCP & 1.50 & 1.22 \\
\hline
\end{tabular}

The contents of triglycerides (TG), total cholesterol (TC), high-density lipid cholesterol (HDL-C), and low-density lipid cholesterol (LDLC) in serum were determined using Automatic Biochemical Analyzer in Animal Health Research Division Lab, Khumaltar. The data obtained were analyzed by Microsoft excel 2007.

\section{Results and Discussion}

The following data shows that Cholesterol has been lowered successively from T1R1-T4R4 in Sakini male and female and Giriraja breed respectively. This lowering mechanism is due to bile salt hydrolase (BSH) released from microorganisms. Bacteria of numerous species (mostly probiotics) isolated from gastrointestinal tract express bile salt hydrolase activity. This capacity to express BSH activities help to resist the toxicity of conjugated bile acid (TDCA). The ability of probiotic strains to hydrolyze bile salts often been included among the criteria for probiotic strain selection and a number of bile salt hydrolases have been indentified and characterized.

Oral administration of probiotics of cholesterol solubility and thereby, absorption of cholesterol levels as much as $22-30 \%$ as shown in Fig 1 and 2. Deconjugated bile salts are less efficiently reabsorbed than their conjugated counterparts, which results in the excretion of larger amounts of free bile salts in feces. Also, free bile salts are less efficient in the solubilization and absorption of lipids in the gut. Therefore, deconjugation of bile salts could lead to reduction in serum cholesterol either by increasing the demand for cholesterol for de novo synthesis of bile acids to replace in feces or by reducing the intestinal lumen. Probiotics have been widely assessed for their effects on lipid profiles such as total cholestrol, LDLcholesterol, HDL- cholesterol and triglycerides. The trial group of Giriaja and Sakini bred in the premises of Swine and Avian Research Program upto to the production line, serum evaluation was done which concluded that probiotics fed with varied concentration had lower Cholesterol although the bsh gene for Bile Salt Hydrolase expression would additionally uplift the gravity.

It is therefore that with haphazard use of antibiotics for growth and mitigating the pathogen, Probiotics would help to erase all the problems. Certain strains of probiotics has the property to lower the blood level of LDL or bad cholestrol with the help of $\mathrm{BSH}$. Supplementation of diet with Probiotics has the potential to reduce serum cholesterol levels. 


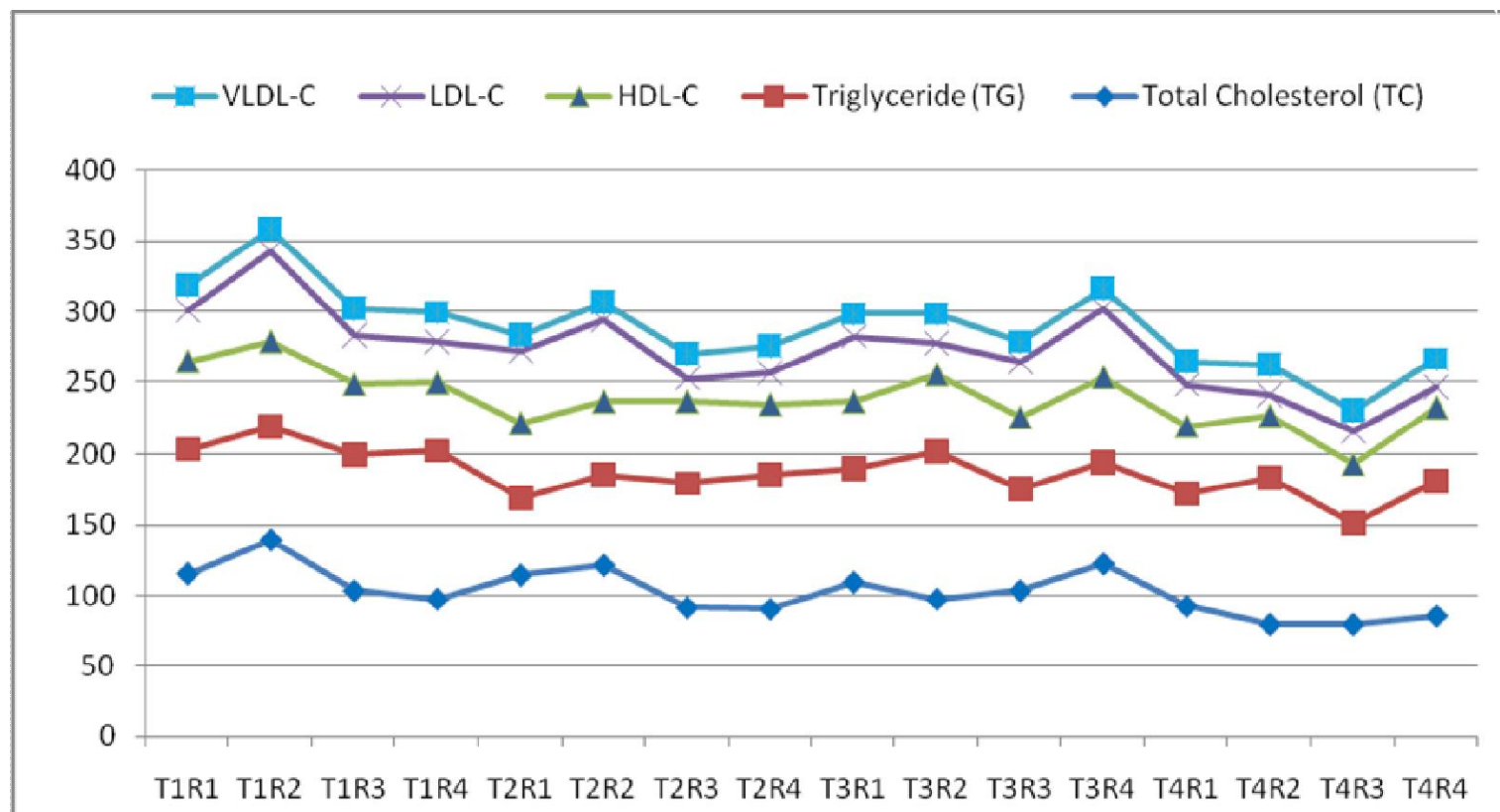

A

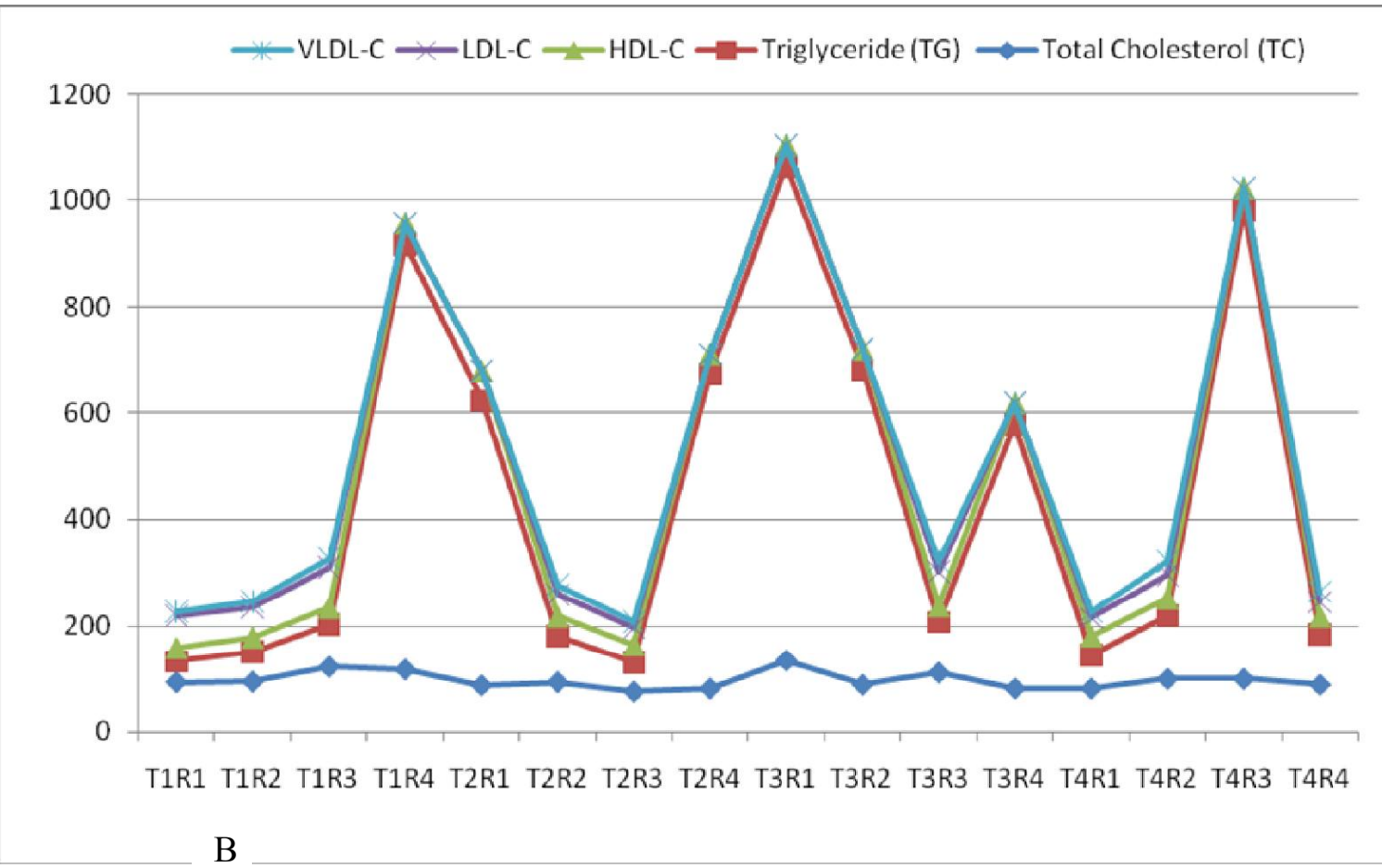

Fig1. Illustration of Cholesterol Lowering properties of (A) Sakini Male and (B) Female respectively 
Neupane et al. (2017) Bang. J. Anim. Sci. 46 (4):225-229

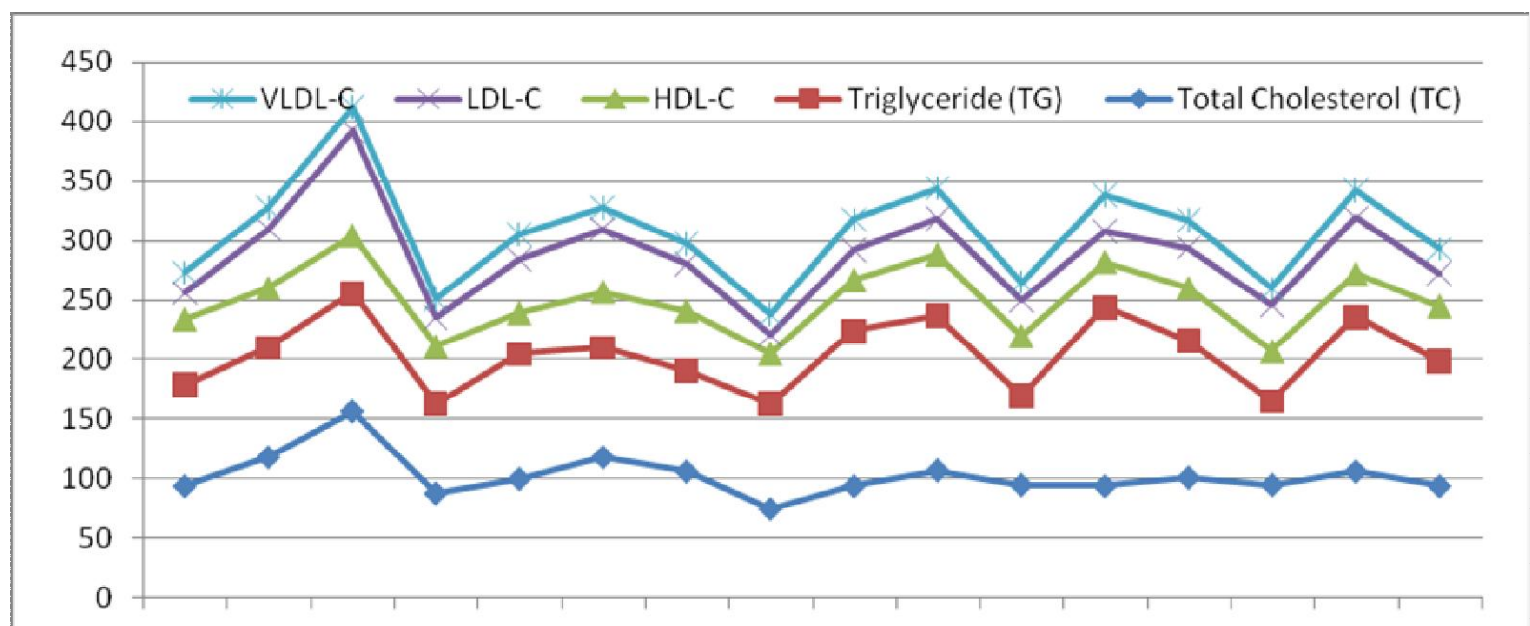

T5R1 T5R2 T5R3 T5R4 T6R1 T6R2 T6R3 T6R4 T7R1 T7R2 T7R3 T7R4 T8R1 T8R2 T8R3 T8R4

A

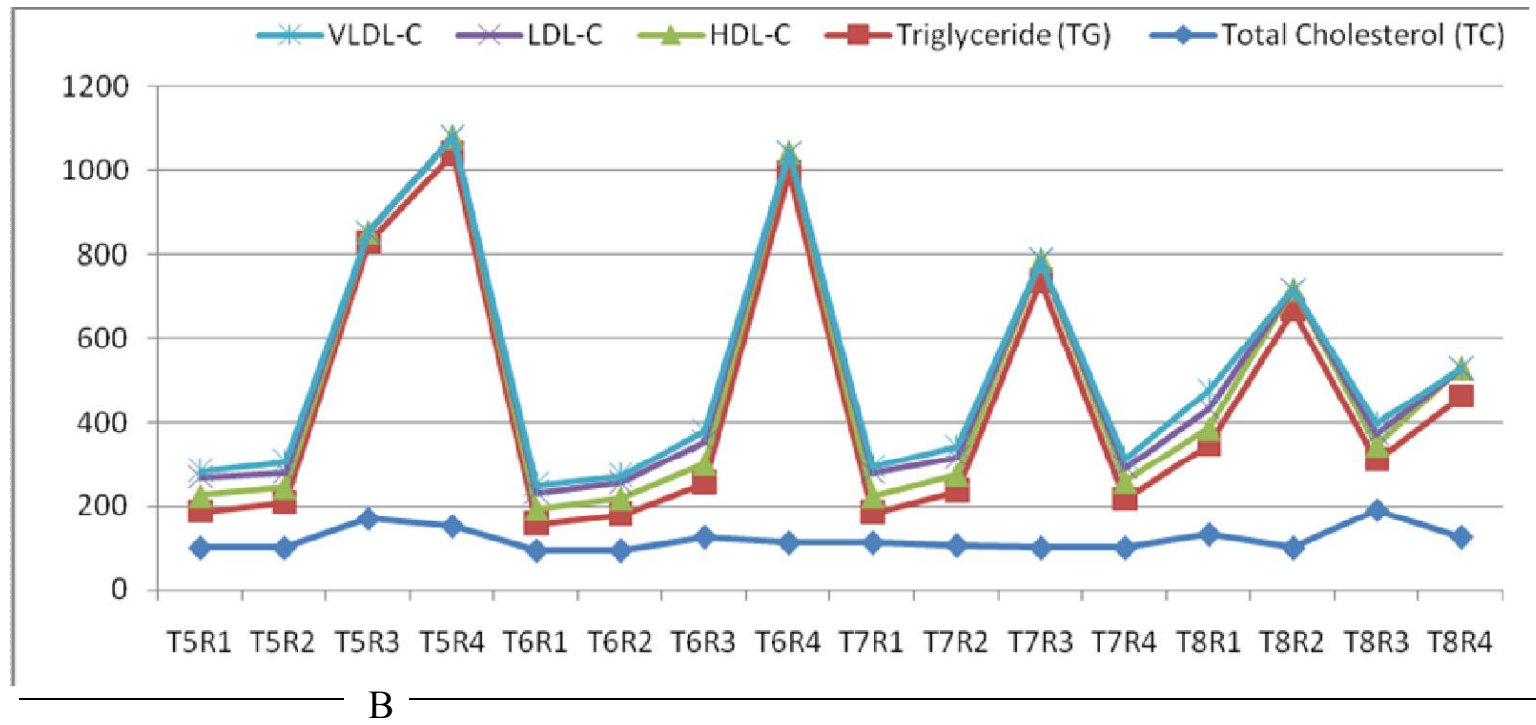

Fig 2. Illustration of Cholesterol Lowering properties of (A) Giriraja Male and (B) Female respectively

\section{Conclusion}

Many of the proposed mechanisms and experiment evidence specifically targeting cholesterol-lowering effects remain controversial. Thus, more properly-designed in vivo trails may disclose additional understanding and knowledge to eliminate the controversies, to better understand the underlying mechanisms and for better safety assessment prior to consumption.

\section{Acknowledgement}

The technical committee of Agriculture and Forestery University (AFU) is gratefully acknlwledged. The Swine an Avian Reserch Program of Khumaltar, Lalitpur is also highly acknowledged.

\section{References}

Bhurtel R and BKP Shaha (2000). Poultry development in Nepal, constraints and potentialities. Research report 45 . Winrock International, 51pp.

CBS (2016). Nepal Commercial poultry survey, 2071/072. Central Bureau of Statistics. Government of Nepal.

Chiang SS and TM Pan (2012). Beneficial effects of Lactobacillus paracasei subsp. paracasei 
NTU 101 and its fermented products. Applied Microbiology and Biotechnology 93(3):90316

Fuller (1989). Probiotics in man and animals. The Journal of Applied Bacteriology 66(5):365378.

Lye HS, CY Kuan, JA Ewe, WY Fung and MT Liong (2009). The improvement of hypertension by probiotics: effects on cholesterol, diabetes, renin, and phytoestrogens. Internastion Journal of Molecular Science 10:3755-75.
Shrestha SB (2000). Production performance of Australorp hens. In: Proceeding of forth National Workshop on Livestock and Fisheries Research, 24-26 April, 2000, Pakhribas, pp. 65-71.

WHO (2009). Cardiovascular Disease; Fact sheet N³17, Geneva, Switzerland, September, 2009. Available at: http://www.who.int/mediacentre/factsheets/f s317/en/print.html (accessed on 19 May2010). 